\title{
Der Herausgabeanspruch gemäss Art. 75 E-FIDLEG
}

\author{
Nicolas Dommer *
}

Voraussichtlich Mitte 2019 wird das neue Finanzdienstleistungsgesetz in Kraft treten, das unter anderem den Schutz der Kunden von Finanzdienstleistern bezweckt. Hierzu wird den Kunden in Art. 75 E-FIDLEG ein jederzeitiger Anspruch auf Herausgabe einer Kopie ihres Dossiers sowie sämtlicher weiterer sie betreffende Dokumente, die der Finanzdienstleister im Rahmen der Geschäftsbeziehung erstellt hat, eingeräumt. Seinen Zweck, eine Lücke im Kundenschutz zu schliessen, erfüllt dieser vorgesehene Herausgabeanspruch indessen nur dann, wenn die auftragsrechtlichen Bestimmungen auf das Verhältnis zwischen Kunde und Finanzdienstleister nicht anwendbar sind. Ansonsten gewährt Art. 400 OR dem Kunden bereits heute einen weitergehenden, jedoch kostenpflichtigen Informationsanspruch.

I. Einleitung 222

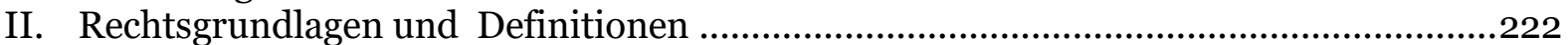

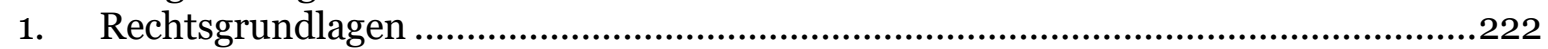

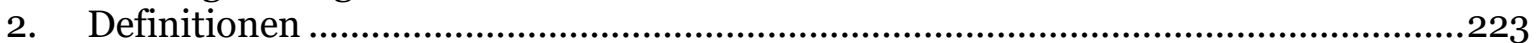

3. Abgrenzung zu Art. 8 DSG und Art. 400 OR ..........................................................224

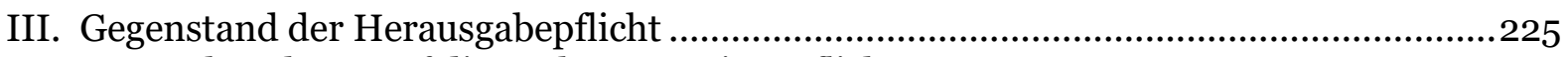

1. Beschränkung auf die Dokumentationspflicht gemäss Art. 17 E-FIDLEG................225

2. Gegenstand der Dokumentationspflicht gemäss Art. 17 E-FIDLEG ..........................227

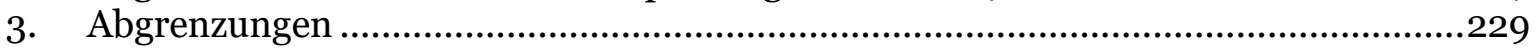

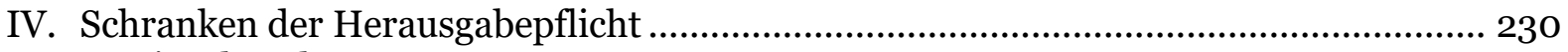

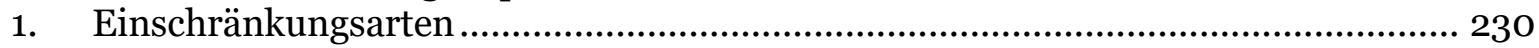

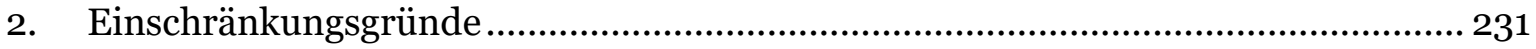

3. Verzicht auf Herausgabe von Dokumenten .............................................................232

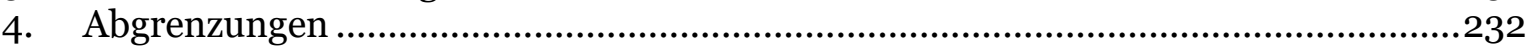

V. Modalitäten des Herausgabebegehrens...............................................................233

1. Folgen des mangelhaften Begehrens …....................................................................233

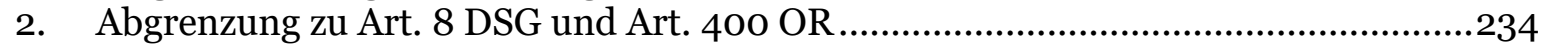

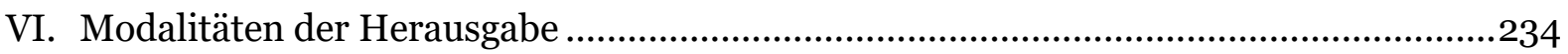

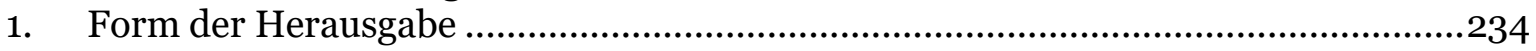

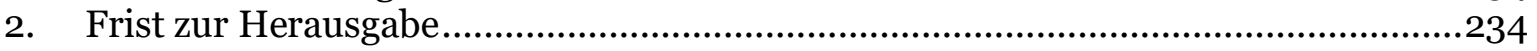

3. Kosten der Herausgabe ........................................................................................234

4. Prozessuale Durchsetzung des Herausgabeanspruchs ...............................................235

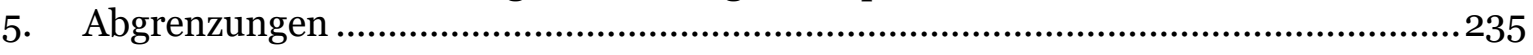

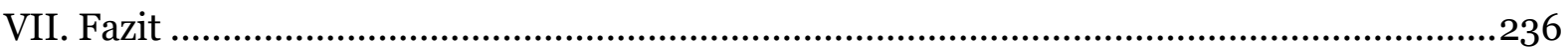

Zitiervorschlag: Nicolas Dommer, Der Herausgabeanspruch gemäss Art. 75 E-FIDLEG, in: sui-generis 2018, S. 221

URL: $\quad$ sui-generis.ch/70

DOI: $\quad$ https://doi.org/10.21257/sg.70

* Nicolas Dommer, Dr. iur., fasst im vorliegenden Beitrag einige Erkenntnisse seiner Dissertation «Die Auskunftspflichten der Bank gegenüber Vermögensverhaltungskunden» (ISBN: 978-3o3751-989-9) zusammen.

Dieses Werk ist lizenziert unter einer Creative Commons Namensnennung - Weitergabe unter gleichen Bedingungen 4.0 International Lizenz. 


\section{Einleitung}

1 Das Finanzdienstleistungsgesetz, das voraussichtlich frühestens Mitte 2019 in Kraft treten wird, soll insbesondere den Schutz von Kundinnen und Kunden ${ }^{1}$ von Finanzdienstleistern gewährleisten (Art. 1 E-FIDLEG ${ }^{2}$ ). Ein Mittel, um dies zu erreichen, ist, die Geltendmachung zivilrechtlicher Ansprüche $\mathrm{zu}$ erleichtern (Art. 1 Abs. 2), was wiederum die genügende Kenntnis der Geschäfts- und Rechtsbeziehung voraussetzt. Diese Kenntnis erlangt der Kunde, indem ein ausreichender Informationsfluss zwischen ihm und dem Finanzdienstleister sichergestellt wird3. Mit Art. 75 soll dem Kunden deshalb ein Anspruch auf die ihn betreffenden Dokumente eingeräumt werden 4.

Im vorliegenden Aufsatz werden der Gegenstand, die Schranken sowie die Modalitäten des Herausgabeanspruchs gemäss Art. 75 und 76 E-FIDLEG untersucht und dargelegt, inwiefern sich dieser Herausgabeanspruch vom Auskunftsanspruch gemäss Art. 8 DSG5 sowie dem Anspruch auf Rechenschaftsablegung gemäss Art. $400 \mathrm{OR}^{6}$ unterscheidet. Hierzu werden vorab der Wortlaut von Art. 75 und

1 Aus Gründen der besseren Lesbarkeit wird im Folgenden auf die gleichzeitige Verwendung männlicher und weiblicher Sprachformen verzichtet. Sämtliche Personenbezeichnungen gelten gleichermassen für beiderlei Geschlecht.

2 Entwurf vom Dezember 2015 für ein Bundesgesetz über die Finanzdienstleistungen.

3 Botschaft vom 4. November 2015 zum Finanzdienstleistungsgesetz (FIDLEG) und zum Finanzinstitutsgesetz (FINIG) (BBl 2015 8901), S. 8940 .

4 Botschaft (Fn. 3), BBl 20158924.

5 Bundesgesetz vom 19. Juni 1992 über den Datenschutz (SR 235.1).

6 Bundesgesetz vom 30. März 2011 betreffend die Ergänzung des Schweizerischen Zivilgesetzbuches (Fünfter Teil: Obligationenrecht; SR 220).
76 E-FIDLEG wiedergegeben und die relevanten Begriffe definiert.

\section{Rechtsgrundlagen und Definitionen}

\section{Rechtsgrundlagen}

3 Der Herausgabeanspruch von Kunden gegenüber dem Finanzdienstleister wird in den Art. 75 und 76 E-FIDLEG, die vom Parlament unverändert verabschiedet wurden7, wie folgt geregelt:

\section{«Art. 75 Anspruch}

${ }^{1}$ Die Kundin und der Kunde haben jederzeit Anspruch auf Herausgabe einer Kopie ihres Dossiers sowie sämtlicher weiterer sie betreffenden Dokumente, die der Finanzdienstleiser im Rahmen der Geschäftsbeziehung erstellt hat.

2 Mit Einverständnis der Kundin oder des Kunden kann die Herausgabe in elektronischer Form erfolgen.

\section{Art. $76 \quad$ Verfahren}

1 Wer einen Anspruch geltend machen will, stellt schriftlich oder in anderer durch Text nachweisbarer Form ein entsprechendes Gesuch.

2 Der Finanzdienstleister lässt der Kundin oder dem Kunden innert 30 Tagen nach Erhalt des Gesuchs unentgeltlich eine Kopie der betreffenden Dokumente zukommen.

$7 \longdiv { \text { Beschluss des Ständerats vom 14. Dezember } 2 0 1 6 }$ und Beschluss des Nationalrats vom 13. September 2017 (15.073). 
3 Kommt er dem Gesuch auf Herausgabe nicht nach, so kann die Kundin oder der Kunde das Gericht anrufen.

4 Eine allfällige Weigerung des Finanzdienstleisters zur Herausgabe kann in einem späteren Rechtsstreit vom zuständigen Gericht beim Entscheid über die Prozesskosten berücksichtigt werden.»

\section{Definitionen}

\section{a) Kundendossier (Dossier)}

4 Der Gegenstand der Herausgabepflicht knüpft an den Begriff des Kundendossiers an, ohne dass dieser Begriff im Gesetzesentwurf definiert wäre. In der Botschaft wird klärend festgehalten, dass von der Herausgabepflicht «sowohl Dokumente oder Unterlagen in Papierform als auch entsprechende elektronische oder elektronisch geführte Dokumente, Dateien oder Aufzeichnungen aller Art [erfasst werden], soweit es sich dabei um die massgebenden Informationen und Dokumentationen handelt, zu deren Führung der Finanzdienstleister nach Massgabe von Artikel 17 verpflichtet ist» 8 .

5 Weiter heisst es, «dass lediglich rein interne Dokumente wie vorbereitende Studien, Notizen oder (Vertrags-)Entwürfe, bezüglich welcher gerade keine Informations- oder Dokumentationspflicht besteht und die daher für die Überprüfung des vertrags- und gesetzeskonformen Verhaltens des Finanzdienstleisters nicht relevant sind»9, von der Herausgabepflicht ausgenommen sind. Dies bedeu-

\footnotetext{
Botschaft (Fn. 3), BBl 20158995.
}

Botschaft (Fn. 3), BBl 20158995. tet, dass sich der Herausgabeanspruch gemäss dem Willen des Gesetzgebers auf die nach Art. 17 E-FIDLEG zu dokumentierenden Informationen beschränkt ${ }^{10}$, da lediglich diese Angaben relevant seien für die Überprüfung des vertrags- und gesetzeskonformen Verhaltens des Finanzdienstleisters, was dem Zweck des Herausgabeanspruchs entspricht.

6 Eine weitergehende Definition des Begriffs «Kundendossier» erübrigt sich vor diesem Hintergrund. Es ist allerdings nicht nachvollziehbar, wieso der Bezug zwischen Art. 17 und Art. 75 E-FIDLEG nur in der Botschaft und nicht auch im Gesetz hergestellt wurde.

\section{b) Den Kunden betreffende Dokumente}

7 Die Definition der weiteren, den Kunden betreffenden Dokumente, die der Finanzdienstleister im Rahmen seiner Geschäftsbeziehung erstellt hat und die gemäss Art. 75 E-FIDLEG ebenfalls der Herausgabepflicht unterliegen, ist obsolet. Auch hier gilt, dass sich der Gegenstand der Herausgabepflicht mit jenem der Dokumentationspflicht nach Art. 17 E-FIDLEG deckt ${ }^{11}$. Die Dokumente, die den Kunden betreffen, können also nicht über die im Rahmen der Dokumentationspflicht zu erstellenden Dokumente hinausgehen.
10 Sethe Rolf/Seiler Moritz, Dokumentation und Rechenschaft im geplanten FIDLEG, in: Waldburger/Sester/Peter/Baer (Hrsg.), Law \& Economics - Festschrift für Peter Nobel zum 70. Geburtstag, Bern 2015, S. 431 ff., Fn. 28.

11 Botschaft (Fn. 3), BBl 20158995. 


\section{c) Verhältnis von Art. $75 \mathrm{zu}$ den Art. 17 und 18 E-FIDLEG}

8 Obwohl die der Herausgabepflicht unterliegenden Dokumente mit der Dokumentation gemäss Art. 17 E-FIDLEG gleichzusetzen sind, sieht die Botschaft in Art. 75 E-FIDLEG eine Ergänzung zu Art. 17 und 1812. Art. 18 Abs. 1 verpflichtet den Finanzdienstleister, dem Kunden eine Kopie der Dokumentation nach Art. 17 zuzustellen. Im Entwurf des Bundesrats war noch vorgesehen, dass die Zustellung dieser Dokumentation spontan zu erfolgen hat ${ }^{13}$. Das Parlament hat Art. 18 Abs. 1 aber dahingehend angepasst, dass der Finanzdienstleister seinen Kunden bloss auf deren Wunsch hin eine Kopie der Dokumentation nach Art. 17 zuzustellen hat ${ }^{14}$. Aus gesetzessystematischer Sicht hat das Parlament damit meines Erachtens einen Fehler begangen. In seiner ursprünglichen Fassung hätte Art. 18 Abs. 1 wohl bezweckt, dass der Finanzdienstleister seinem Kunden die Dokumentation gemäss Art. 17 sofort nach deren Erstellung hätte zukommen lassen oder zumindest zugänglich machen müssen, um diesem die unmittelbare Kontrolle darüber zu ermöglichen. Fehler hätten so früher erkannt und korrigiert werden können. In der jetzigen Ausgestaltung unterscheidet sich Art. 18 Abs. 1 aus Kundensicht meines Erachtens nicht mehr wesentlich von Art. 75 .

9 Der einzige Unterschied zwischen Art. 18 Abs. 1 und Art. 75 E-FIDLEG liegt nun-

12 Botschaft (Fn. 3), BBl 20158995.

13 Dommer Nicolas, Die Auskunftspflichten der Bank gegenüber Vermögensverwaltungskunden, Diss. Universität St. Gallen, St. Gallen 2018, N 341.

14 Beschluss des Ständerats vom 14. Dezember 2016 und Beschluss des Nationalrats vom 13. September 2017 (15.073). mehr in deren Rechtsnatur. Art. 75 gewährt dem Kunden einen privatrechtlichen Anspruch auf eine Kopie der Dokumentation ${ }^{15}$, währenddem Art. 17 und 18 öffentlich-rechtlicher Natur sind. Die Einhaltung von Art. 17 und 18 wird entsprechend durch die Aufsichtsbehörde geprüft ${ }^{16}$. Ein Kunde, der ein aufsichtsrechtliches Verfahren gegen den Finanzdienstleister anstrebt, weil dieser seinen Pflichten gemäss Art. 17 und 18 nicht nachgekommen ist, erlangt in diesem Verfahren jedoch keine Parteistellung ${ }^{17}$. Er kann die Erfüllung dieser aufsichtsrechtlichen Pflichten des Finanzdienstleisters somit nicht direkt geltend machen $^{18}$. Den privatrechtlichen Anspruch nach Art. 75 hingegen kann der Kunde selbst vor Gericht durchsetzen (Art. 76 Abs. 3) ${ }^{19}$. Dies führt meines Erachtens dazu, dass Art. 18 Abs. 1 nur noch dann zur Anwendung gelangt, wenn der Finanzdienstleister seinen Herausgabepflichten nach Art. 75 nicht nachgekommen ist und der Kunde zusätzlichen Druck ausüben möchte, indem er die Aufsichtsbehörde entsprechend informiert. Betreffend die herauszugebenden Dokumente unterscheiden sich Art. 75 und Art. 18 Abs. 1 nach der hier vertretenen Ansicht nämlich nicht.

\section{Abgrenzung zu Art. 8 DSG und Art. 400 OR}

10 Der Herausgabeanspruch gemäss Art. 75 E-FIDLEG «tritt kumulativ zu den bestehenden vertraglichen und gesetzlichen Informationsansprüchen und zivilpro-

15 Botschaft (Fn. 3), BBl 20158995.

16 Botschaft (Fn. 3), BBl 20158921.

17 BGE 139 II 279 E. 4; Botschaft (Fn. 3), BBl 2015 8921.

18 Botschaft (Fn. 3), BBl 20158941.

19 Vgl. auch Botschaft (Fn. 3), BBl 20158941. 
zessualen Editionspflichten hinzu» ${ }^{20}$. Die auskunftsersuchende Person bedient sich jener rechtlichen Grundlage, die in der entsprechenden Situation am passendsten für sie ist ${ }^{21}$.

\section{Gegenstand der Herausgabepflicht}

\section{Beschränkung auf die} Dokumentationspflicht gemäss Art. 17 E-FIDLEG

11 Wie bereits ausgeführt, ist der Gegenstand der Herausgabepflicht gemäss Art. 75 E-FIDLEG nach dem Willen des Gesetzgebers jenem der Dokumentationspflicht von Art.17 gleichzusetzen. Art. 75 gewährt dem Kunden eines Finanzdienstleisters somit einen jederzeitigen, privatrechtlichen Anspruch auf die gemäss Art. 17 zu erstellenden Dokumente. Diese Auslegung wird auch von der Systematik des E-FIDLEG gestützt. Art. 75 erfüllt vor allem dort seinen Zweck, wo Art. 400 OR nicht anwendbar ist $^{22}$. In diesen Fällen gewährt das E-FIDLEG dem Kunden einen privatrechtlichen Informationsanspruch, den er bis anhin nicht hatte.

Der jederzeitige Anspruch auf eine Kopie der Dokumentation gemäss Art. 17 E-FIDLEG, der dem Kunden in Art. 75 eingeräumt wird, ermöglicht ihm einen besseren Einblick in die vom Finanzdienstleister vorgenommene Dienstleis-

20 Reichart Peter/Meyer Richard, Klagen gegen Finanzdienstleister im FIDLEG-Entwurf - eine kritische Würdigung, in: SZW 2016, S. 390 ff., 396; Botschaft (Fn. 3), BBl 20158995.

${ }_{21}$ Sethe/Seiler (Fn. 10), S. 447.

22 Das ist bspw. beim reinen Verkauf von Finanzinstrumenten der Fall; vgl. Sethe/Seiler (Fn. 10), S. 443 f., mit einer Aufzählung der Vertragsverhältnisse ohne Auftragskomponente, die dem E-FIDLEG unterstellt sind. tungserbringung. Das führt $\mathrm{zu}$ einer Stärkung der Position des Kunden in einem Rechtsstreit mit dem Finanzdienstleister ${ }^{23}$. Es werden die Voraussetzungen für eine effektive Rechtsdurchsetzung geschaffen ${ }^{24}$. Ein Anspruch, der über die im Rahmen der Dokumentationspflicht zu erstellenden Dokumente hinausgeht, ist nicht erforderlich. Der Kunde ist bei Vertragsverhältnissen mit dem Finanzdienstleister, die keine Auftragskomponente aufweisen, nämlich weniger schutzbedürftig25. Dem wurde bei der Ausgestaltung der vertraglichen Informationsrechte Rechnung getragen, indem für die Gewährung von Informationsrechten nach Vertragstyp unterschieden wurde. Bei einem Kauf- oder Werkvertrag besteht bspw. nicht das gleiche legitime Bedürfnis nach Information wie bei einem Auftrag, der die Wahrung fremder Interessen bezweckt ${ }^{26}$. Dem Käufer bzw. dem Besteller werden deshalb nicht dieselben Informationsansprüche eingeräumt wie dem Auftraggeber. Diese Wertungen werden mit der Einführung von Art. 75 E-FIDLEG nicht komplett aufgegeben.

13 Der Informationsanspruch von Art. 400 OR geht weiter als der in Art. 75 E-FIDLEG vorgesehene Anspruch ${ }^{27}$. Dennoch ist der Herausgabeanspruch nach Art. 75 genügend umfangreich, um dem Kunden, der keinen auftragsrechtlichen Informationsanspruch hat, die Überprüfung des vertrags- und gesetzes-

23 Schleiffer Patrick/Schärli Patrick, Ein Überblick über das künftige Finanzdienstleistungsgesetz und Finanzinstitutsgesetz, in: GesKR 2014, S. 334 ff., 338.

24 Botschaft (Fn. 3), BBl 20158995.

25 Sethe/Seiler (Fn. 10), S. 444.

26 Reichart/Meyer (Fn. 20), S. 396 f.

27 Dommer (Fn. 13), N 355 ff. 
konformen Verhaltens des Finanzdienstleisters $^{28}$ - und damit eine effektive Rechtsdurchsetzung - zu ermöglichen 29. Zusätzlich erhöht wird der Informationsfluss zwischen Kunde und Finanzdienstleister durch die Informations- und die Rechenschaftspflicht (Art. 9 f. bzw. Art. 18), die das E-FIDLEG dem Finanzdienstleister auferlegt. Die Systematik des Gesetzes stützt damit die Beschränkung der Herausgabepflicht nach Art. 75 auf die Dokumente, die gemäss Art. 17 erstellt werden müssen.

Das Gleiche gilt für die gesetzesübergreifende Systematik. Die Beschränkung des Herausgabeanspruchs auf die gemäss Art. 17 E-FIDLEG zu dokumentierenden Angaben bedeutet, dass der Kunde gestützt auf Art. 75 keinen Anspruch auf die im Rahmen der Rechenschaftsablegung gemäss Art. 18 Abs. 2 Bst. b und c erstellten Dokumente hat ${ }^{3}$. Bei diesen Dokumenten handelt es sich bspw. um Kontound Depotauszüge oder Performanceanalysen ${ }^{31}$. Art. 75 gewährt dem Kunden, entgegen dem Wortlaut der Bestimmung, auch dann keinen Anspruch auf diese Dokumente, wenn sie im Kundendossier enthalten sind. Von der gesetzesübergreifenden Systematik her ist dies deshalb begrüssenswert, weil Art. 75

(Fn. 3), BBl 2015 8995; vgl. auch Rz. 4

29 Vgl. Schleiffer/Schärli (Fn. 23), S. 338; Botschaft (Fn. 3), BBl 20158995.

30 Die Informationen, über die gemäss Art. 18 Abs. 2 Bst. a E-FIDLEG Rechenschaft abgelegt werden muss, sind auch nach Art. 17 Abs. 1 zu dokumentieren und deshalb Gegenstand der Herausgabepflicht nach Art. 75.

31 A.M. wohl Wyss Lukas, Mehrparteienverfahren und kollektiver Rechtsschutz vor Zivilgerichten in der Schweiz, in: Jusletter, publiziert am 16. Februar 2015, N 100, gemäss dem auch Kontoauszüge, Vermögensausweise und Aufzeichnungen der Bank über Kundenkontakte, wie Telefon- und Gesprächsnotizen, Gegenstand der Herausgabepflicht gemäss Art. 75 E-FIDLEG sind. einen kostenlosen und jederzeitigen Anspruch auf diese Dokumente gewähren würde.

15 Mit Art. 400 OR existiert aber bereits eine privatrechtliche Grundlage, die dem Kunden einen jederzeitigen Anspruch auf dieselben Dokumente einräumt. Der Beauftragte darf dort allerdings eine Entschädigung für die Ausfertigung und $\mathrm{Zu}-$ stellung der Kopien verlangen ${ }^{2}$, was meines Erachtens sachgerecht ist. Das jederzeitige Recht, die Dokumente, über die bereits gemäss Art. 18 Abs. 2 Bst. b und c E-FIDLEG Rechenschaft abzulegen ist, gestützt auf Art. 75 erneut und kostenlos verlangen zu können, wäre nach der hier vertretenen Ansicht ein Eingriff in die vertragliche Regelung, der nur dazu führen würde, dass der Finanzdienstleister die entsprechenden Dokumente für den Kunden aufzubewahren hätte. Der Kunde wäre nicht mehr daran interessiert, bspw. die Konto- und Depotauszüge selbst aufzubewahren, wenn er diese jederzeit, kostenlos und wiederholt vom Finanzdienstleister verlangen könnte. Dies wäre aber nicht im Sinne des Gesetzgebers, der mit der Einführung von Art. 75 die Geltendmachung zivilrechtlicher Ansprüche erleichtern und nicht den Aufbewahrungsaufwand des Kunden reduzieren möchte.

16 In den Fällen, in denen Art. 400 OR auf das Verhältnis zwischen dem Kunden und dem Finanzdienstleister nicht anwendbar ist und mit Art. 75 E-FIDLEG eine Lücke im Kundenschutz geschlossen wird, findet keine periodische Rechen-

$32 \overline{\text { Jörg Florian S./Arter Oliver, Herausgabe- und }}$ Rechenschaftspflicht des unabhängigen Vermögensverwalters - Übergabepflichten richten sich nach dem Auftragsrecht, in: ST 2004, S. 297 ff., 300. 
schaftsablegung gemäss Art. 18 Abs. 2 E-FIDLEG statt, da es sich um Rechtsverhältnisse handelt, die mit einmaliger Ausführung erledigt sind33. Der Schutz dieser Kunden wird bei einer Beschränkung des Herausgabeanspruchs auf die gemäss Art. 17 zu erstellenden Dokumente somit nicht tangiert.

Schliesslich ist festzustellen, dass das Parlament eine Änderung von Art. 18 Abs. 2 E-FIDLEG beschlossen hat und damit ein wenig in die oben beschriebene Systematik eingegriffen hat. Die Bestimmung sieht nun vor, dass der Finanzdienstleister auf Anfrage der Kundinnen und Kunden und nicht mehr spontan Rechenschaft abzulegen hat. Damit räumt sie dem Kunden einen meines Erachtens jederzeitigen und kostenlosen Anspruch auf Rechenschaftsablegung ein. Im Unterschied $\mathrm{zu}$ Art. 400 OR ist der Anspruch jedoch öffentlich-rechtlicher $\mathrm{Na}$ tur, weshalb sich die Durchsetzung für den Kunden schwieriger gestaltet 34 . Zudem kann die Änderung von Art. 18 Abs. 2 E-FIDLEG im Gegensatz zur beschlossenen Anpassung von Abs. 1 damit begründet werden, dass das E-FIDLEG dem Kunden denselben Anspruch nicht bereits in Art. 75 einräumt. Gleichzeitig stützt die Anpassung des Parlaments die vorliegend vertretene Ansicht, wonach sich der Gegenstand von Art. 75 E-FIDLEG auf die Dokumentation gemäss Art. 17 beschränkt, da der Kunde mit Art. 18 Abs. 2 E-FIDLEG und

33 Vgl. Sethe/Seiler (Fn. 10), S. 443 f. mit Ausführungen $\mathrm{zu}$ den Vertragsverhältnissen zwischen Kunde und Finanzdienstleister ohne Auftragskomponente; siehe auch Eggen Mirjam, Finanzprodukte - Auftrag oder Kauf?, in: SZW 2011, S. 625 ff., 628 f., mit einer Aufzählung der Execution-only-Geschäfte und deren rechtlicher Qualifikation.

34 M.w.H. Rz. 9.
Art. 400 OR bereits einen jederzeitigen öffentlich- und privatrechtlichen Anspruch auf Rechenschaftsablegung hat.

\section{Gegenstand der Dokumentations- pflicht gemäss Art. 17 E-FIDLEG}

18 Im Folgenden wird der mögliche Gegenstand der Dokumentationspflicht umschrieben. Da Art. 17 diesbezüglich etwas vage ist, werden nach Inkrafttreten des E-FIDLEG wohl noch konkretisierende Bestimmungen erlassen, seien es Verordnungen oder Selbstregulierung, um hier Rechtssicherheit zu schaffen35. Mit der in diesem Kapitel dargestellten Dokumentation wird der Pflicht gemäss Art. 17 nach der hier vertretenen Ansicht aber Genüge getan.

\section{a) Mit dem Kunden vereinbarte Finanzdienstleistungen}

19 Gemäss Art. 17 Abs. 1 Bst. a E-FIDLEG sind die vereinbarten Finanzdienstleistungen zu dokumentieren. Die möglichen Finanzdienstleistungen, die vereinbart werden können, werden in Art. 3 Bst. d definiert. Die Dokumentation der vereinbarten Finanzdienstleistung wird in Form eines schriftlichen Vertrags erfolgen. Dies ist bereits jetzt Standard und bspw. hinsichtlich des Vermögensverwaltungsvertrags im FINMA-Rundschreiben betreffend die Eckwerte zur Vermögensverwaltung sowie den Richtlinien für Vermögensverwaltungsaufträge so vorgesehen ${ }^{6}$.

35 Gassmann Rochus, Dokumentationspflicht des Finanzdienstleisters - und bei Unklarheiten fragen Sie Ihren Richter; in: Böhme/Gähwiler/ Simoni/Zuberbühler (Hrsg.), Ohne jegliche Haftung - Festschrift für Willi Fischer - Beiträge zum schweizerischen Haftpflicht- und Schuldrecht, Zürich/Basel/Genf 2016, S. 143 ff., 146.

36 FINMA, Rundschreiben 2009/1 betreffend Eckwerte zur Vermögensverwaltung vom 1. Januar 


\section{b) Über den Kunden erhobene Informationen}

20 Der Finanzdienstleister hat die über den Kunden erhobenen Informationen in geeigneter Weise zu dokumentieren (Art. 17 Abs. 1 Bst. a E-FIDLEG). Nach der hier vertretenen Ansicht handelt es sich dabei um die im Rahmen der Angemessenheits- oder Eignungsprüfung erhobenen Angaben und um das Ergebnis dieser Prüfung (Art. 12 bzw. 13)37. Ansonsten findet sich im E-FIDLEG keine weitere Bestimmung, die den Finanzdienstleister zur Erhebung von Informationen über ihre Kunden verpflichtet. Eine Pflicht, Informationen $\mathrm{zu}$ dokumentieren, die nicht aufgrund des E-FIDLEG zu erheben sind, vermag Art. 17 meines Erachtens nicht $\mathrm{zu}$ statuieren. Aus diesem Grunde bilden bspw. Informationen, die gemäss der VSB 1638 zur Identifizierung des Kunden zu erheben sind, nicht Gegenstand der Dokumentationspflicht nach E-FIDLEG und sind somit auch nicht Gegenstand der Herausgabepflicht gemäss Art. 75. Dies schmälert den Kundenschutz indes nicht. Über diese Informationen kann der Kunde gestützt auf Art. 8 DSG Auskunft verlangen39.

\section{c) Hinweise gemäss Art. 17 Abs. 1 Bst. b E-FIDLEG}

21 Gewisse Finanzdienstleister haben gemäss Art. 14 Abs. 1 E-FIDLEG für die Erbringung ihrer Dienstleistungen weder eine Angemessenheits- noch eine Eignungsprüfung durchzuführen. Über diesen Umstand haben sie den Kunden vor

2009, N 8; Richtlinien für Vermögensverwaltungsaufträge der Schweizerischen Bankiervereinigung vom 1. März 2017 (VVRL), Art. 2.

37 Vgl. auch Botschaft (Fn. 3), BBl 20158959.

38 Vereinbarung vom 1. Juni 2015 über die Standesregeln zur Sorgfaltspflicht der Banken.

39 Dommer (Fn. 13), N 71 ff. der Dienstleistungserbringung zu informieren und dies gemäss Art. 17 Abs. 1 Bst. b zu dokumentieren. Falls der Finanzdienstleister eine Angemessenheitsoder Eignungsprüfung durchführen muss, die Informationen, die er dazu erhält, für deren Durchführung aber nicht ausreichen, hat er den Kunden vor der Erbringung der Dienstleistung darauf hinzuweisen (Art. 16). Dass dieser Hinweis stattgefunden hat, ist gemäss Art. 17 Abs. 1 Bst. b zu dokumentieren. Gemäss demselben Buchstaben ist auch zu dokumentieren, dass dem Kunden von der Erbringung einer Dienstleistung abgeraten wurde, wenn der Finanzdienstleister zur Auffassung gelangt, dass ein bestimmtes Finanzinstrument für diesen nicht geeignet ist.

\section{d) Für den Kunden erbrachte Finanzdienstleistungen}

22 Was Finanzdienstleistungen sind, die gemäss Art. 17 Abs. 1 Bst. c E-FIDLEG zu dokumentieren sind, wird in Art. 3 Bst. d geregelt. Meines Erachtens genügt es aber nicht, bspw. bloss zu dokumentieren, dass für den Kunden ein bestimmtes Finanzinstrument erworben wurde (Art. 3 Bst. d Ziff. 1). Vielmehr müssen analog zu Art. 38 FinfraG40 die getätigten Geschäfte mit allen Angaben dokumentiert werden, die für deren Nachvollziehbarkeit erforderlich sind.

\section{e) Dokumentation gemäss Art. 17 Abs. 2 E-FIDLEG}

23 Art. 17 Abs. 2 E-FIDLEG wurde vom Parlament angepasst. Die Finanzdienstleister wurden bei der Vermögensverwaltung

40 Bundesgesetz über die Finanzmarktinfrastrukturen und das Marktverhalten im Effekten- und Derivatehandel vom 19. Juni 2015 (SR 958.1). 
von der Pflicht zur Dokumentation der Bedürfnisse der Kunden sowie der Gründe für jede Empfehlung, die zum Erwerb, zum Halten oder zur Veräusserung eines Finanzinstruments führt, befreit. Diese Dokumentationspflicht ist nur noch bei der Anlageberatung vorgesehen ${ }^{41}$. Dies ist insofern zu begrüssen, da im reinen Vermögensverwaltungsverhältnis keine Empfehlungen gemacht werden. Der Finanzdienstleister entscheidet selbst, ob er für den Kunden ein Finanzinstrument erwirbt, hält oder veräussert. Es wäre aus Kundensicht jedoch wünschenswert gewesen, die Finanzdienstleister bei der Vermögensverwaltung dazu zu verpflichten, die Gründe, die zum Erwerb, zum Halten oder zur Veräusserung eines Finanzinstruments geführt haben, zu dokumentieren ${ }^{42}$.

\section{f) Aufbewahrungsfrist der Dokumentation}

24 Die Dokumente, die im Rahmen der Dokumentationspflicht gemäss Art. 17 E-FIDLEG zu erstellen sind, sind «unter Berücksichtigung der obligationenrechtlichen Dokumentations- und Aufbewahrungspflichten»43 während mindestens zehn Jahren aufzubewahren44. Der Herausgabeanspruch, der dem Kunden in Art. 75 eingeräumt wird, kennt jedoch keine zeitliche Beschränkung45. Wenn der Finanzdienstleister die Dokumente über die gesetzliche Aufbewahrungsfrist

$4 1 \longdiv { \text { Beschluss des Ständerats vom 14. Dezember } 2 0 1 6 }$ und Beschluss des Nationalrats vom 13. September 2017 (15.073).

42 Vgl. Dommer (Fn. 13), N $363 \mathrm{f}$.

43 Botschaft (Fn. 3), BBl 20158960.

44 Botschaft (Fn. 3), BBl 20158960.

45 Ein Vorstoss von Ständerat Peter Föhn (SVP/SZ) zur Beschränkung des Herausgabeanspruchs auf die gesetzlich vorgesehene Aufbewahrungsfrist von zehn Jahren wurde vom Parlament abgelehnt (AB 2016 S 1170). hinaus aufbewahrt, hat er bei einem Herausgabebegehren des Kunden Kopien davon herauszugeben.

\section{Abgrenzungen}

\section{a) Zu Art. 8 DSG}

25 Die Auskunftspflicht nach Art. 8 DSG geht teilweise weiter als die Herausgabepflicht gemäss Art. 75 E-FIDLEG und umgekehrt. Der Autor des vorliegenden Artikels vertritt die Ansicht, dass bloss Personendaten Gegenstand der Auskunftspflicht gemäss Art. 8 DSG sind und nicht Kopien von Originaldokumenten, die an gewissen Stellen auch Personendaten enthalten 46 . Da Art. 75 E-FIDLEG einen Anspruch auf Kopien der Dokumentation gemäss Art. 17 gewährt und diese nicht zwangsläufig ausschliesslich Personendaten des Kunden enthält, ist der Informationsanspruch nach Art. 75 in dieser Hinsicht umfassender als jener von Art. 8 DSG. Finanzdienstleister bearbeiten aber auch Personendaten, die nicht Gegenstand der Dokumentationspflicht von Art. 17 E-FIDLEG sind47, über die sie gemäss Art. 8 DSG aber Auskunft zu erteilen haben. Zudem hat der Kunde gemäss Art. 8 Abs. 2 DSG Anspruch auf Auskunft über die Herkunft der Daten, den Zweck und gegebenenfalls die Rechtsgrundlagen des Bearbeitens, die Kategorien der bearbeiteten Personendaten, die an der Sammlung Beteiligten und die Datenempfänger. Diese Informationen erhält der Kunde gestützt auf Art. 75 E-FIDLEG nicht.

46 Vgl. Dommer (Fn. 13), N 185 ff.

47 Dommer (Fn. 13), m.w.H. zum Gegenstand der Auskunftspflicht nach Art. 8 DSG, N 69 ff. 


\section{b) Zu Art. 400 OR}

Den beauftragten Finanzdienstleister trifft bereits gestützt auf Art. 400 OR eine Dokumentationspflicht48. Der Inhalt dieser Dokumentation wird vom Gegenstand der Rechenschaftspflicht bestimmt49 und umfasst alles, was von Relevanz ist, um den Kunden über die Auftragsausführung aufzuklären50. Die Dokumentationspflicht nach Art. 400 OR ist damit umfassender als jene nach Art. 17 E-FIDLEG51. Entsprechend ist auch der Rechenschaftsanspruch des Kunden gemäss Art. 400 OR umfassender als sein Herausgabeanspruch nach Art. 75 E-FIDLEG52. Ihm unterliegen bspw. auch die Korrespondenz53 oder die Aufzeichnungen von Kundenkontakten54. Zudem hat der Beauftragte im Rahmen der Rechenschaftsablegung einen Bericht abzufassen, der «über alle wesentlichen Vorgänge des konkreten Auftrags und die Erläuterung ihrer Bedeutung»55 Auf-

48 Sethe Rolf, in: Schäfer/Sethe/Lang (Hrsg.), Handbuch der Vermögensverwaltung in Deutschland, Österreich, der Schweiz und Liechtenstein, 2. Aufl., München/Wien/Basel 2016, § 31 N 12.

49 Hafner Stefan, Die Rechenschaftspflicht des Beauftragten - Ein Beitrag zum Informationsrecht im Auftragsvertrag, Diss. Universität St. Gallen, Berlin 2007, S. 84.

5o Fellmann Walter, Der einfache Auftrag, in: Heinz Hausheer (Hrsg.), Berner Kommentar zum Schweizerischen Privatrecht, 6. Band, 2. Abteilung, 4. Teilband, Bern 1992, Art. 400 OR N 19.

51 Dommer (Fn. 13), N 355 ff.

52 Vgl. Fischer Philipp/Amiguet Antoine, LSFIN/ LEFIN - «Wind of Change» ou "Highway to Hell»?, in: Anwaltsrevue 2015, S. 35 ff., 37, die davon ausgehen, dass Art. 75 E-FIDLEG keine weitergehenden Ansprüche gewährt als das geltende Recht, insbesondere nicht als Art. 400 OR, Art. 8 DSG oder Art. 158 ZPO.

53 Dommer (Fn. 13), N 246 f. und 249.

54 Wobei der Inhalt von Kundenkontakten auch der Herausgabe von Dokumenten gemäss Art. 75 E-FIDLEG unterliegen kann. Das ist insbesondere bei den gemäss Art. 17 Abs. 1 Bst. b zu dokumentierenden Informationen der Fall.

55 Fellmann, Art. 400 OR N 27. schluss gibt. Auf solche Erklärungen haben Kunden von Finanzdienstleistern gemäss Art. 75 E-FIDLEG keinen Anspruch. Es müssen ihnen einzig Kopien von Dokumenten herausgegeben werden 56 .

27 Sofern das Vertragsverhältnis zwischen Kunde und Finanzdienstleister den auftragsrechtlichen Regeln untersteht, hat die Einführung von Art. 75 i.V.m. Art. 17 E-FIDLEG hinsichtlich des Gegenstands somit lediglich eine Doppelung der schon bestehenden Dokumentations- und Informationspflichten des Finanzdienstleisters zur Folge, wobei die Pflicht zur Rechenschaftsablegung gemäss Art. 400 OR über den Herausgabenspruch gemäss Art. 75 E-FIDLEG hinausgeht.

\section{Schranken der Herausgabepflicht}

\section{Einschränkungsarten}

28 Als Einschränkungsart kommt für die Herausgabepflicht gemäss Art. 75 E-FIDLEG einzig die Verweigerung in Betracht. Dies ergibt sich, wie sich zeigen wird, aus den limitierten Gründen, die eine Einschränkung der Herausgabepflicht erlauben. Aus den möglichen Gründen der Einschränkung ergibt sich zudem, dass die Herausgabepflicht nicht eingeschränkt werden muss, wenn die Voraussetzungen dafür erfüllt sind, sondern kann.

29 Den Entscheid, die Herausgabe zu verweigern, hat der Finanzdienstleister meines Erachtens zu begründen, da der Kunde ansonsten das Gericht anrufen (Art. 76 Abs. 3) und das Gericht in einem späteren Rechtsstreit die Verweigerung

56 Vgl. Sethe/Seiler (Fn. 10), S. 440. 
der Herausgabe beim Entscheid über die Prozesskosten berücksichtigen kann (Art. 76 Abs. 4).

\section{Einschränkungsgründe}

\section{a) Geheimhaltungsinteressen}

30 Dadurch, dass der Gegenstand der Herausgabepflicht nach Art. 75 E-FIDLEG der Dokumentation gemäss Art. 17 entspricht57, ist eine Einschränkung der Herausgabepflicht nur sehr beschränkt möglich. Geheimhaltungsinteressen des Finanzdienstleisters, die eine Einschränkung rechtfertigen würden, sind nicht ersichtlich.

31 Auch allfällige Geheimhaltungsinteressen Dritter, insbesondere das Bankkundengeheimnis, erlauben keine Einschränkung der Herausgabepflicht. Informationen Dritter, die der Herausgabepflicht gemäss Art. 75 E-FIDLEG unterliegen, werden höchstens im Rahmen der Dokumentation der erbrachten Finanzdienstleistungen - und auch hier nur beim Erwerb oder der Veräusserung von Finanzinstrumenten sowie bei der Annahme und Übermittlung von Aufträgen, die Finanzinstrumente zum Gegenstand haben - erfasst. In diesem Zusammenhang wird meines Erachtens die Bezeichnung der Gegenpartei festzuhalten sein. In Analogie zu Art. 1 Abs. 3 FinfraVFINMA5 8 genügt nach der hier vertretenen Ansicht aber die Dokumentation der «Stammnummer mit dem dazugehörenden Namen bzw. eine Identifikation der

57 Vgl. Botschaft (Fn. 3), BBl 20158995.

58 Verordnung der Eidgenössischen Finanzmarktaufsicht vom 3. Dezember 2015 über die Finanzmarktinfrastrukturen und das Marktverhalten im Effekten- und Derivatehandel (SR 958.111).
Abrechnung des Gegenauftrages»59. Das Bankkundengeheimnis greift in diesem Fall nicht, da die Bezeichnung der Gegenpartei ohne Angabe darüber auskommt, bei welcher Bank sie Kunde ist. Sollte dennoch dokumentiert werden, bei welcher Bank die Gegenpartei Kunde ist, darf meines Erachtens von einer stillschweigenden Einwilligung in die Bekanntgabe der Bankverbindung ausgegangen werden ${ }^{60}$.

32 Das Datenschutzgesetz verbietet die Bekanntgabe der Bezeichnung der Gegenpartei an den Kunden ebenfalls nicht. Die Bekanntgabe wird mit dem Herausgabeanspruch des Kunden gemäss Art. 75 E-FIDLEG durch Gesetz gerechtfertigt (Art. 13 Abs. 1 DSG).

\section{b) Rechtsmissbrauchsverbot}

33 Erlaubt ist die Verweigerung der Herausgabe, wenn der Anspruch rechtsmissbräuchlich geltend gemacht wird61. Dies kann nach der hier vertretenen Ansicht bei einer schikanösen Rechtsausübung der Fall sein, die bspw. dann vorliegt, wenn die Herausgabe unangemessen häufig verlangt wird, ohne dass dafür ein berechtigtes Interesse besteht ${ }^{62}$. Analog zu Art. 2 Abs. 1 Bst. a VDSG63 liegt meines Erachtens eine unangemessen häufige Geltendmachung des Herausgabean-

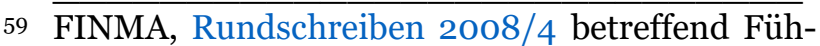
rung des Effektenjournals durch Effektenhändler und Teilnehmer vom 1. Januar 2009, N 40.

60 Vgl. auch Emch Urs/Renz Hugo/Arpagaus Reto, Das Schweizerische Bankgeschäft, 7. Aufl., Zürich/Basel/Genf 2011, N 481.

61 Gl.M. Sethe/Seiler (Fn. 10), S. 439 f.

62 Vgl. Fellmann, Art. 400 OR N 80, der diesen Einschränkungsgrund auch für die Einschränkung der Rechenschaftspflicht nach Art. 400 OR vorsieht.

63 Verordnung vom 14. Juni 1993 zum Bundesgesetz über den Datenschutz (SR 235.11). 
spruchs vor, wenn der Kunde Kopien der Dokumente bereits in den letzten zwölf Monaten vor dem Gesuch einmal erhalten hat und kein schutzwürdiges Interesse an einer erneuten Herausgabe nachweisen kann. Ebenfalls als schikanös kann die Rechtsausübung qualifiziert werden, wenn von einer Organisation zur Geltendmachung des Anspruchs auf Herausgabe aufgerufen wird, um dem zur Herausgabe verpflichteten Finanzdienstleister offensichtlich $\mathrm{zu}$ schaden, und darüber hinaus kein schutzwürdiges Individualinteresse an den verlangten Kopien besteht.

\section{c) Verjährung}

34 Der Herausgabeanspruch gemäss Art. 75 E-FIDLEG ist privatrechtlicher Natur 64 . Er unterliegt deshalb, mangels abweichender Regelung im Gesetzesentwurf, der zehnjährigen Verjährungsfrist nach Art. 127 OR. Diese beginnt mit Beendigung des Vertragsverhältnisses zu laufen. Wie auch beim Rechenschaftsanspruch gemäss Art. 400 OR bedeutet dies, dass der Anspruch nach Vertragsbeendigung bis zum Ablauf der Verjährungsfrist bestehen bleibt ${ }^{65}$.

\section{Verzicht auf Herausgabe von Dokumenten}

Aus Art. 75 Abs. 2 E-FIDLEG, der regelt, dass auf die physische Herausgabe von Kopien lediglich zugunsten einer Herausgabe in elektronischer Form verzichtet werden kann, lässt sich ableiten, dass es sich bei Art. 75 um eine zwingende Bestimmung handelt. Ein vollumfänglicher

64 Botschaft (Fn. 3), BBl 20158995.

65 Fellmann, Art. 400 OR N 99.
Verzicht auf die Rechenschaftsablegung ist nicht vorgesehen 66 .

\section{Abgrenzungen}

\section{a) Zu Art. 8 DSG}

36 Im Gegensatz zur Einschränkung der Herausgabe nach Art. 75 E-FIDLEG, die vorgenommen werden kann, aber nicht muss, besteht für die Auskunft gemäss Art. 8 DSG eine Pflicht zur Einschränkung, wenn die Voraussetzungen dafür erfüllt sind 67 . Auch hinsichtlich der Einschränkungsarten und -gründe unterscheiden sich die beiden Auskunftspflichten. Art. 9 DSG regelt abschliessend und differenziert, wie und weshalb das datenschutzrechtliche Auskunftsrecht eingeschränkt werden muss. Im E-FIDLEG fehlt eine entsprechende Bestimmung. Nach der hier vertretenen Ansicht kann die Herausgabe gemäss Art. 75 E-FIDLEG bloss verweigert und nicht eingeschränkt oder aufgeschoben werden. Als Einschränkungsgründe kommen nur das Rechtsmissbrauchsverbot und die Verjährung in Frage.

\section{b) Zu Art. 400 OR}

37 Die Rechenschaftsablegung nach Art. 400 OR darf, neben dem Rechtsmissbrauchsverbot und der Verjährung, auch gestützt auf Geheimhaltungsinteressen oder Interessen an der Verhältnismässigkeit des Aufwandes eingeschränkt oder verweigert werden 68 . Im Vergleich zur Einschränkung des Herausgabeanspruchs gemäss Art. 75

66 Sethe/Seiler (Fn. 10), Fn. 63.

67 Rosenthal David, in: Rosenthal/Jöhri (Hrsg.), Handkommentar zum Datenschutzgesetz sowie weiteren, ausgewählten Bestimmungen, Zürich 2008, Art. 9 DSG N 3.

68 Dommer (Fn. 13), N $280 \mathrm{ff}$. 
E-FIDLEG stehen dem Finanzdienstleister im Rahmen von Art. 400 OR somit mehr Möglichkeiten zur Verfügung, um die Auskunft einzuschränken.

\section{Modalitäten des Herausgabebegehrens}

38 Der Anspruch auf Herausgabe von Dokumenten kann gemäss dem Wortlaut von Art. 75 Abs. 1 E-FIDLEG jederzeit geltend gemacht werden und besteht unabhängig von weiteren vertraglichen oder gesetzlichen Auskunftsansprüchen sowie von prozessualen Editionspflichten des Finanzdienstleisters 69 . Aus dem Wortlaut von Art. 75 Abs. 1 E-FIDLEG, der dem Kunden einen jederzeitigen Herausgabeanspruch einräumt, lässt sich meines Erachtens ableiten, dass der Anspruch auch wiederholt geltend gemacht werden kann.

39 Das Herausgabebegehren ist gemäss Art. 76 Abs. 1 E-FIDLEG «schriftlich oder in anderer durch Text nachweisbarer Form» zu stellen. Die Regelung des E-FIDLEG ist technikneutral ausgestaltet. Eine elektronische Geltendmachung des Anspruchs auf Herausgabe von Dokumenten ist genauso gültig wie eine Geltendmachung per Brief oder per Fax. Mündlich gestellte Auskunftsbegehren hingegen sind nicht vorgesehen. Dies trägt aus Beweisgründen sowohl zum Schutz des Kunden als auch der Bank bei. Die Möglichkeit eines mündlichen Auskunftsbegehrens entspräche deshalb auch nicht dem in Art. 1 Abs. 1 E-FIDLEG festgelegten Zweck des Finanzdienstleistungsgesetzes.
40 Ein Nachweis der Identität durch den Gesuchsteller wird im Unterschied zum Auskunftsbegehren nach Art. 8 DSG (Art. 1 Abs. 2 Bst. a VDSG) nicht verlangt. Dies ist in der Regel aber auch nicht notwendig, da die Kopien der Dokumente dem Kunden herauszugeben sind, und dieser von der Bank bereits bei der Aufnahme der Vertragsbeziehung identifiziert wurde. Sollten die Kopien der Dokumente allerdings an eine Adresse geliefert werden, die nicht mit jener übereinstimmt, welche die Bank in ihren Systemen erfasst hat, ist ein Identitätsnachweis zu verlangen, um einer Verletzung des Bankkundengeheimnisses vorzubeugen. Dieses greift nur gegenüber dem Kunden selbst oder gegenüber Personen nicht, die von diesem bevollmächtigt wurde ${ }^{70}$.

\section{Folgen des mangelhaften Begehrens}

41 Ein Gesuch um Herausgabe von Kopien von Dokumenten, das nicht «schriftlich oder in anderer durch Text nachweisbarer Form» (Art. 76 Abs. 1 E-FIDLEG) gestellt wird, erfüllt die Formerfordernisse nicht. Der Kunde ist in diesem Fall auf die schriftliche Eingabe zu verweisen.

42 Fehlt lediglich ein Identitätsnachweis, obwohl dieser aufgrund der unbekannten Zustelladresse notwendig wäre, ist das Gesuch aber in durch Text nachweisbarer Form gestellt worden, so erfüllt es die Anforderungen von Art. 76 Abs. 1 E-FIDLEG und kann nicht per se ungültig sein. Die Bank hat in diesem Falle auf

70 Kleiner Beat/Schwob Renate/Winzeler Christoph, Kommentar zum Bundesgesetz über die Banken und Sparkassen - Stand: 22. Nachlieferung 2014, Zobl/Schwob/Geiger/Winzeler/ Kaufmann/Weber/Kramer (Hrsg.), 22. Aufl., Zürich 2014, Art. 47 BankG N 14. 
das Gesuch einzugehen und bei der gesuchstellenden Person den fehlenden Identitätsnachweis einzufordern.

\section{Abgrenzung zu Art. 8 DSG und Art. 400 OR}

43 Die Anforderungen an das Auskunftsbegehren sind für die Geltendmachung des datenschutzrechtlichen Auskunftsrechts etwas höher als für jene des Anspruchs auf Herausgabe von Kopien gemäss Art. 75 E-FIDLEG und des Anspruchs auf Rechenschaftsablage nach Art. 400 OR. Ein elektronisch eingereichtes Gesuch ist gemäss Art. 1 Abs. 2 VDSG nur dann gültig, wenn der Inhaber der Datensammlung dies ausdrücklich vorsieht und angemessene Massnahmen trifft, um die Identifizierung der betroffenen Person sicherzustellen und die Auskunftserteilung vor dem Zugriff unberechtigter Dritter zu schützen. Entsprechende Voraussetzungen für ein elektronisches Gesuch sind weder dem E-FIDLEG noch dem Auftragsrecht zu entnehmen. Zudem ist zur gültigen Geltendmachung des datenschutzrechtlichen Auskunftsrechts, im Gegensatz zum Herausgabeanspruch gemäss Art. 75 E-FIDLEG und dem Anspruch auf Rechenschaftsablage nach Art. 400 OR, immer ein Identitätsnachweis zu erbringen.

\section{Modalitäten der Herausgabe}

\section{Form der Herausgabe}

44 Die Herausgabe der geschuldeten Dokumente hat grundsätzlich physisch in Form einer Kopie zu erfolgen (Art. 75 Abs. 1 E-FIDLEG). Mit dem Einverständnis des Kunden dürfen die Dokumente aber auch in elektronischer Form ausgehändigt werden (Art. 75 Abs. 2).

\section{Frist zur Herausgabe}

45 Gemäss Art. 76 Abs. 2 E-FIDLEG sind dem Kunden die Kopien der Dokumente innert 30 Tagen nach Erhalt des Gesuchs zukommen zu lassen. Eine Regelung, die dem Finanzdienstleister die Erstreckung der Frist erlaubt, ist nicht vorgesehen. Da der Finanzdienstleister aber dazu verpflichtet ist, seine Dokumentation, und damit den Gegenstand der Herausgabepflicht, so zu gestalten, dass er «seiner Rechenschaftspflicht gemäss Art. 18 nachkommen kann und jederzeit fähig ist, der Aufsichtsbehörde oder ihren Beauftragten Auskunft über die erbrachten Dienstleistungen $\mathrm{zu}$ erteilen» 71 , ist dies auch nicht notwendig. Die Herausgabe sollte dem Finanzdienstleister wenig Aufwand bereiten. Es kann ihm deshalb zugemutet werden, den Anspruch innert 30 Tagen zu erfüllen.

46 Die Begründung einer allfälligen Verweigerung der Herausgabe ist dem Kunden in Analogie zu Art. 1 Abs. 4 VDSG meines Erachtens ebenfalls innert 30 Tagen nach Erhalt des Gesuchs zuzustellen.

\section{Kosten der Herausgabe}

47 Die Herausgabe der Dokumente hat unentgeltlich zu erfolgen (Art. 76 Abs. 2 E-FIDLEG). Die Bank hat weder Anspruch auf Entschädigung noch auf Aufwendungsersatz ${ }^{72}$. Damit will der Gesetzgeber verhindern, dass der Herausgabeanspruch des Kunden mit Kostenrisiken und -folgen belastet wird. Dies vor dem Hintergrund, dass der Herausgabeanspruch eine Massnahme darstellt, welche die Geltendmachung zivilrechtlicher An-

71 Botschaft (Fn. 3), BBl 2015 8959; so auch Bühler Christoph B., «Suitability \& Appropriateness»: Was ist wirklich neu?, in: GesKR 2016, S. 1 ff., 10.

72 Botschaft (Fn. 3), BBl 20158996. 
sprüche erleichtern und gerade auch die (Prozess-)Kostenrisiken vermindern soll73.

\section{Prozessuale Durchsetzung des Herausgabeanspruchs}

48 Kommt der Finanzdienstleister seiner Herausgabepflicht nicht nach, so kann der Kunde das Gericht anrufen (Art. 76 Abs. 3 E-FIDLEG). Die ZPO74 soll diesbezüglich um Art. 251a ergänzt werden75. Dieser Artikel sieht vor, dass für die Durchsetzung des Herausgabeanspruchs das summarische Verfahren zur Anwendung gelangt ${ }^{76}$. Damit will der Gesetzgeber eine möglichst speditive Durchsetzung gewährleisten 77 .

\section{Abgrenzungen}

\section{a) Zu Art. 8 DSG}

49 Im Gegensatz zu Art. 75 E-FIDLEG gewährt Art. 8 DSG der auskunftsersuchenden Person nach Ansicht des Autors keinen Anspruch auf Kopien von Originaldokumenten, sondern lediglich einen Anspruch auf Personendaten ${ }^{78}$, die bspw. in einer Liste zusammengefasst werden können. Von dieser Liste ist dem Kunden eine Kopie zuzustellen.

Der Inhaber der Datensammlung ist befugt, die 30-tägige Frist, in der die Auskunft gemäss Art. 8 DSG zu erteilen ist, zu erstrecken (Art. 1 Abs. 4 VDSG). Unter Umständen darf er von der auskunftser-

\footnotetext{
Botschaft (Fn. 3), BBl 20158996.

74 Schweizerische Zivilprozessordnung vom 19. Dezember 2008 (SR 272).

75 Beschluss des Ständerats vom 14. Dezember 2016 und Beschluss des Nationalrats vom 13. September 2017 (15.073).

76 Botschaft (Fn. 3), BBl 20159007.

77 Botschaft (Fn. 3), BBl 20159007.

78 Dommer (Fn. 13), N 185 ff.
}

suchenden Person auch eine Kostenbeteiligung verlangen (Art. 2 VDSG). Diese Möglichkeiten sieht das E-FIDLEG nicht vor. Eine solche wäre aber auch nicht im Sinne des Gesetzes, das eine effektive Rechtsdurchsetzung mit geringen Kostenrisiken ermöglichen will79.

51 Sollte der Inhaber der Datensammlung seiner Auskunftspflicht gar nicht nachkommen, kann der Kunde diesen Anspruch gemäss Art. 243 Abs. 2 Bst. d ZPO im vereinfachten Verfahren durchsetzen. Für die Durchsetzung des Herausgabeanspruchs nach Art. 75 E-FIDLEG ist hingegen das summarische Verfahren vorgesehen (Art. 251a E-ZPO ${ }^{80}$ ).

\section{b) Zu Art. 400 OR}

52 Auch im Rahmen der Rechenschaftsablegung gemäss Art. 400 OR ist der Auftraggeber befugt, Kopien von Originaldokumenten zu verlangen, allerdings hat er dafür die Kosten zu tragen (Art. 402 Abs. 1 OR) ${ }^{81}$. Die erneute Rechenschaftsablegung ist, im Unterschied zur Herausgabe nach Art. 75 E-FIDLEG, ebenfalls kostenpflichtig82. Soweit die Ansprüche gemäss Art. 400 OR und Art. 75 E-FIDLEG deckungsgleich sind, können mit der Geltendmachung des Herausgabeanspruchs gemäss Art. 75 E-FIDLEG anstelle der Rechenschaftsablegung nach Art. 400 OR also Kosten vermieden werden, die unter geltendem Recht noch anfallen. Der Kunde hat zum Beispiel die

79 Botschaft (Fn. 3), BBl 20158995.

8o Entwurf vom Dezember 2015 für die Änderung der schweizerischen Zivilprozessordnung vom 19. Dezember 2008 (SR 272).

81 Hofstetter Joseph, Der Auftrag und die Geschäftsführung ohne Auftrag, in: Wiegand (Hrsg.), Schweizerisches Privatrecht, 7. Band, 6. Teilband, Obligationenrecht - Besondere Vertragsverhältnisse, Basel 2000, S. 1 ff., 118.

82 Hofstetter (Fn. 81), S. 118. 
Möglichkeit, gestützt auf Art. 400 OR einen Rechenschaftsbericht zu verlangen, der über alle wesentlichen Vorgänge Aufschluss gibt und deren Bedeutung erläutert ${ }^{83}$. Statt gestützt auf diesen Bericht Kopien von Dokumenten zu verlangen, wofür er die Kosten zu tragen hätte, kann er seinen Herausgabeanspruch gemäss Art. 75 E-FIDLEG geltend machen. Überdies gestaltet sich die prozessuale Durchsetzung des Herausgabeanspruchs gemäss Art. 75 E-FIDLEG einfacher, da das summarische Verfahren zur Anwendung gelangt (Art. 251a E-ZPO). Der Rechenschaftsanspruch nach Art. 400 OR muss im ordentlichen bzw. vereinfachten Verfahren durchgesetzt werden 84 .

53 Hinsichtlich der Frist zur Beantwortung des Auskunftsbegehrens unterscheiden sich Art. 75 E-FIDLEG und Art. 400 OR ebenfalls. Der Finanzdienstleister als Beauftragter hat rechtzeitig Rechenschaft abzulegen. Ob eine Rechenschaftsablegung, die erst 30 Tage nach Erhalt des Gesuchs erfolgt, wie das in Art. 76 Abs. 2 E-FIDLEG vorgesehen ist, noch rechtzeitig ist, ist im Einzelfall zu entscheiden ${ }^{85}$.

\section{Fazit}

54 Das Ziel des Gesetzgebers, mit Art. 75 E-FIDLEG den Informationsfluss zwischen Finanzdienstleister und Kunde sicherzustellen und dadurch eine Lücke im Kundenschutz zu schliessen, wurde meines Erachtens nur teilweise erreicht. Dies liegt vor allem daran, dass es kaum

$83 \overline{\text { Fellmann, Art. } 400 \text { OR N 27; für die Abfassung }}$ dieses Berichts darf der Beauftragte, mangels anderer Abmachung, eine Entschädigung verlangen.

84 Emmenegger Susan/Good Rahel, 2. Teil Handels-, Wirtschafts- und Immaterialgüterrecht/Aktuelle Fragen des Bankrechts, in: Aktuelle Anwaltspraxis 2013, S. 943 ff., 954.

85 Hofstetter (Fn. 81), S. 118. eine Lücke $\mathrm{zu}$ schliessen galt. Immer dann, wenn das Verhältnis zwischen Finanzdienstleister und Kunde den auftragsrechtlichen Bestimmungen unterliegt, und entsprechend Art. 400 OR zur Anwendung gelangt, ist nämlich für einen ausreichenden Informationsfluss gesorgt. Die Ausführungen haben gezeigt, dass der Auskunftsanspruch gemäss Art. 400 OR sogar umfassender ist als jener von Art. 75 E-FIDLEG, der sich auf die gemäss Art. 17 E-FIDLEG zu dokumentierenden Angaben beschränkt. Die einzigen erkennbaren Vorteile des Herausgabeanspruchs von Art. 75 E-FIDLEG gegenüber dem Rechenschaftsanspruch nach Art. 400 OR liegen für den Kunden in dessen Kostenlosigkeit sowie der erleichterten prozessualen Durchsetzung, was zu einer gewissen Stärkung seiner Position führt.

55 Die wesentliche Leistung von Art. 75 E-FIDLEG beschränkt sich allerdings auf den marginalen Bereich, in dem die auftragsrechtlichen Bestimmungen auf das Verhältnis zwischen Finanzdienstleister und Kunde keine Anwendung finden. In diesem Fall vermag der Herausgabeanspruch eine Lücke im Kundenschutz zu schliessen. 